similar series of rashes varying from the scarlatiniform, morbilliform, lichenoid and urticarial rashes to eruptions resembling erythema multiforme and erythema nodosum.

That the individual mycides are in no way related to any particular group of fungi is generally recognized, and the literature now contains innumerable examples of almost every type of fungus, whether of animal or human origin, trichophyton or epidermophyton producing any variety of mycide. All that one can say is that the greater the inflammatory disturbance in the primary infection the more pronounced and widespread will be the allergic eruption.

\title{
CASE OF PRIMARY CARCINOMA OF THE LUNG.
}

\author{
By A. J. COKKINIS, M.B., F.R.c.s., \\ Assistant Director, The Surgical Unit, St. Mary's Hospital.
}

History.-Male, aged 4I, labourer. Quite well until four months before admission, when he developed a cough and shortness of breath on exertion. The dyspnoa became progressively worse until, a fortnight ago, he had to give up work. Lately he has complained of some pain in his upper right chest, and has observed increasing weakness and loss of weight. There has been a slight spitting up of blood on two or three occasions in the last month. His voice has been getting increasingly husky.

Examination.-Patient shows signs of recent wasting. He breathes with some effort and his voice is weak and husky. The head, neck and upper limbs are markedly cyanosed and congested, with slight œedematous swelling. There is striking evidence of obstruction of the superior vena cava; all the veins of the neck, head, upper limbs and upper trunk are dilated and stand out, forming a very complete pattern of the venous tree.

A hard and fixed mass can be seen and felt in the right supraclavicular region. This has all the characters of carcinomatous lymph-glands. Laryngoscopy shows a right-sided abductor palsy. Both pupils are normal and react to light.

Examination of the chest reveals advanced changes in the upper lobe of the right lung. It is completely dull on percussion, the air entry is very poor and breath-sounds can hardly be heard.

Discussion.-This is clearly a case of carcinoma involving the upper lobe of the right lung. It may have started in the lung tissue, or it may be primarily a carcinoma of the bronchus. Radiography shows a definite opacity corresponding to the area of clinical involvement.

The striking and suggestive clinical features are: $(a)$ The short history; $(b)$ the cough and increasing dyspnœa; $(c)$ the marked venous engorgement pointing to compression of the superior vena cava; $(d)$ the mass of malignant glands in the right supraclavicular region ; $(e)$ the laryngeal abductor palsy, pointing to involvement of the right recurrent laryngeal nerve (probably by the glandular metastasis); $(t)$ the very definite physical signs in the chest. 